\title{
Expression of the ELAV-like protein HuR in human prostate carcinoma is an indicator of disease relapse and linked to COX-2 expression
}

\author{
SILVIA NIESPOREK ${ }^{1 *}$, GLEN KRISTIANSEN $^{1 *}$, ANDREA THOMA $^{1}$, WILKO WEICHERT $^{1}$, \\ AURELIA NOSKE ${ }^{1}$, ANN-CHRISTIN BUCKENDAHL ${ }^{1}$, KLAUS JUNG ${ }^{2}$, \\ CARSTEN STEPHAN $^{2}$, MANFRED DIETEL $^{1}$ and CARSTEN DENKERT ${ }^{1}$ \\ ${ }^{1}$ Institute of Pathology, ${ }^{2}$ Department of Urology, Charité Universitätsmedizin Berlin, Germany
}

Received August 16, 2007; Accepted October 5, 2007

\begin{abstract}
The human ELAV-like protein HuR is involved in the stabilization of the mRNAs of a group of genes implicated in the regulation of cellular growth, angiogenesis and rapid inflammatory response. HuR is a nuclear shuttling protein, translocating bound mRNAs from the nucleus to the cytoplasm. We have previously observed an increased expression of cyclooxygenase-2 (COX-2) in prostate cancer while cell culture studies have shown that HuR stabilizes the mRNA of COX-2. Based on these mechanistic data, we aimed to investigate the role of HuR in prostate cancer by a tissue-based analysis combined with functional evaluation using a cell culture approach. Investigating 104 primary prostate carcinomas by immunohistochemistry, we found HuR expression to be shifted from a nuclear staining in normal prostate glands to a cytoplasmic staining in carcinoma tissue $(\mathrm{p}<0.0001)$. Cytoplasmic HuR expression was significantly correlated with COX-2 expression ( $\mathrm{p}=0.005)$. Loss of nuclear HuR expression was an indicator of earlier PSA-relapse both in univariate $(p=0.04)$ and multivariate survival analysis $(p=0.04)$. HuR inhibition by Leptomycin $\mathrm{B}$ reduced the inducibility of COX-2 in PC-3 prostate cancer cells. We found that the subcellular localization of $\mathrm{HuR}$ is deregulated in a subset of prostate carcinomas, and that this deregulation is linked to an altered expression of the tumorigenic COX-2 protein as well as to an adverse patient prognosis. Our results point to a potential prognostic role of $\mathrm{HuR}$ expression in prostate cancer diagnostics and propose $\mathrm{HuR}$ as a future therapeutic target in prostate cancer therapy.
\end{abstract}

Correspondence to: Dr S. Niesporek, Institute of Pathology, Charité Universitätsmedizin Berlin, Campus Mitte, Charitéplatz 1, D-10117 Berlin, Germany

E-mail: silvia.niesporek@charite.de

${ }^{*}$ Contributed equally

Key words: HuR, cyclooxygenase-2, prostate cancer, prognosis

\section{Introduction}

In Western countries, prostate adenocarcinoma is the most common malignancy in men (1). Radical prostatectomy is the standard therapy in localized disease, but nevertheless, in a subset of cases the disease follows an aggressive course and the patients suffer from disease-relapse, metastasis and cancer-related death. For these patients, novel therapeutic options in addition to the established application of radiotherapy and anti-androgens are needed. The identification of molecular markers deregulated in prostate cancer or linked to unfavourable outcome contributes to the evaluation of potential novel therapeutic targets, which may help to individualize and ameliorate therapy in high-risk patients. Furthermore, established prognostic factors as pre-operative prostate-specific antigen (PSA) level, histological grade, tumor stage, patient age and resection margins are not always sufficient to predict patient outcome. Additional prognostic markers are thus needed to assess the individual prognostic profile and to plan individualized therapy.

The human ELAV-like protein HuR has recently gained attention in cancer research because it regulates the mRNA stability of many growth-promoting genes (2). HuR is a member of the Hu family of RNA-binding proteins and is ubiquitously expressed in many cell types. HuR recognizes and binds mRNAs containing adenosine and uracil- (AU-) rich elements (ARE). These elements are frequently found in the 3 ' untranslated regions of several unstable transcripts encoding cytokines, cell-cycle regulators or proto-oncogenes (2). mRNA binding by HuR stabilizes the mRNA and prevents its rapid degradation by exonucleases (2). The mechanism by which HuR stabilizes RNAs is not completely understood. Functional studies suggest that its cytoplasmic localization might be relevant for its mRNA stabilizing function $(3,4)$. In resting cells $\mathrm{HuR}$ is predominantly localized in the nucleus and translocates to the cytoplasm upon stimulation. As increased cytoplasmic HuR expression has been detected in human malignant tumors, a deregulated HuR pathway has been suggested to be implicated in cancer biology by promoting an abnormal expression of several proteins (5-7).

HuR has also been shown to stabilize the mRNA of cyclooxygenase-2 (COX-2), which contains several AREs 
$(8,9)$. COX-2 is the inducible key-enzyme in the synthesis of prostaglandins (10), and in vitro studies, which demonstrated the implication of COX-2 in prostate cancer cell proliferation and invasiveness (11) underlined the role of COX-2 in this malignancy. We and others have observed an overexpression of COX-2 in prostate cancer $(12,13)$, which was associated with an unfavorable disease outcome in some studies $(14,15)$. Yet, information about the causes of COX-2 overexpression in cancer is still sparse.

In this study, we investigated the hypothesis that a deregulation of HuR expression may contribute to the development and progression of prostate cancer, and thus determined the expression pattern of HuR normal and neoplastic prostate tissue as well as its relation to clinicopathological parameters and patient prognosis. We further evaluated whether a deregulation of HuR expression might add to the increased expression of COX-2 and combined the tissue-based analysis with functional evaluation in a cell culture model.

\section{Patients and methods}

Study population. One hundred and four patients (mean age 63 years, range 47-72 years) who underwent radical prostatectomy for prostate adenocarcinoma at the Charite University Hospital and whose tumors had been investigated for COX-2 expression in a previous study (12) were included in this study (approved by the ethics committee of the Charite University Hospital). Data on pre-operative PSA levels were available for 96 patients $(92.3 \%$, range $0.6-40.6 \mathrm{ng} / \mathrm{ml}$, median $8.6 \mathrm{ng} / \mathrm{ml})$. Clinical follow-up data on PSA values were available for all patients. The median follow-up time of patients who did not experience a PSA-relapse was 46 months. Thirty-six patients (34.6\%) experienced a PSA-relapse. As our cohort comprised patients who had undergone a radical prostatectomy and had no distant metastasis, PSA-relapse was defined strictly (serum PSA $>0.04 \mathrm{ng} / \mathrm{ml}$ ), however, the definition of a PSA-relapse varies considerably among different institutions. Further clinicopathological parameters of the study cohort are given in Table II.

Immunohistochemistry. For immunohistochemistry, we used a monoclonal anti-human HuR antibody (clone 3A2, Santa Cruz Biotechnology, Santa Cruz, CA, 1:500). Staining procedures were performed according to standard protocols as described previously (12).

The intensity of the cytoplasmic and nuclear immunostaining in tumor cells was evaluated independently by two investigators (G. Kristiansen and C. Denkert), in a blinded manner regarding patient characteristics and outcome. Cases with a disagreement were discussed using a multi-headed microscope until consensus was achieved. Prostatectomy specimens were evaluated for staining in non-neoplastic and cancerous prostatic epithelium. The cytoplasmic and the nuclear staining patterns of HuR were evaluated separately, each according to the percentage of positive cells and the intensity of staining. The percentage of positive cells was scored as: 0 (0\%); 1 (1-10\%); 2 (11-50\%); 3 (51-80\%); 4 $(>80 \%)$. The staining intensity was scored as: 0 (negative), 1 (weak), 2 (moderate) and 3 (strong). The whole tissue section was evaluated and average staining intensity and percentage of stained cells were estimated. For the immunoreactivity score (IRS) the percentage of positive cells and staining intensity were multiplied, resulting in a value between 0 and 12. To separate cases with weak or strong expression of cytoplasmic and nuclear HuR we combined cases with an IRS of 0-6 to one group with negative to low HuR expression (HuR-negative), while cases with an IRS of 7-12 were combined into a 'HuR-positive' group (Fig. 1).

Statistical analysis. The statistical significance of correlations between the expression of $\mathrm{HuR}$ and several clinicopathological parameters was assessed by Fisher's exact test or $\chi^{2}$ test. Correlations between the immunoreactivity scores of nuclear and cytoplasmic $\mathrm{HuR}$ as well as of COX-2 expression were calculated using Spearman's rho rank order correlation. The probability of differences in relapse-free survival was determined by the Kaplan-Meier method and the log-rank test. Multivariate survival analysis was performed with the Cox model of proportional hazards using stepwise forward inclusion of variables. Generally, p-values $<0.05$ were considered as significant. For statistical evaluation SPSS software Version 13.0 was used.

Cell lines and HuR inhibition. The human prostate carcinoma cell line PC-3 was obtained from the American Type Culture Collection (Rockville, MD, USA). LNCAP and DU-145 were purchased from the German Collection of Microorganisms and Cell Cultures (DSMZ, Braunschweig, Germany). Cells were cultured in DMEM supplemented with $10 \%$ fetal bovine serum. For induction of COX-2, cells were stimulated 24 h with $10 \mathrm{nM}$ phorbol ester (TPA, Sigma, St. Louis, MO) or $5 \mathrm{ng} / \mathrm{ml}$ interleukin (IL)-1 (R\&D Systems, Minneapolis, $\mathrm{NM}$ ) for $24 \mathrm{~h}$. For pharmacological inhibition of HuR, 5 or $10 \mathrm{ng} / \mathrm{ml}$ Leptomycin B (Sigma) was added $30 \mathrm{~min}$ before stimulation. All experiments were performed in triplicates.

Immunoblotting. Immunoblotting was performed as decribed previously (16). For the detection of COX-2 protein a monoclonal anti-COX-2 antibody (Cayman, USA, 1:500), was used.

\section{Results}

Expression of HuR in human prostate carcinoma and normal prostate tissue. To determine whether HuR expression was altered in neoplastic prostate tissue, we compared the HuR expression patterns between prostate carcinomas and adjacent non-neoplastic prostate glands (Fig. 1). In normal tissue cytoplasmic HuR expression was frequently absent (IRS 0 in $84.6 \%)$. In cancer higher immunoreactivity scores for cytoplasmic HuR were frequent. Only 33 cases $(31.7 \%)$ had an IRS of 0 , while 20 cases $(28.8 \%)$ reached an IRS of 4 , and 19 cases $(18.3 \%)$ reached an IRS of $>6$ and were scored positive. Thus, cytoplasmic HuR expression was significantly increased in cancer $(\mathrm{p}<0.0001$, Table I). Moderate to strong intensity of nuclear HuR expression in $>80 \%$ of cells was detectable in most cases of normal and neoplastic prostate tissue. Thus, in normal tissue an IRS of 8 was reached in 26 cases $(25 \%)$ cases and an IRS of 12 was reached in 57 cases (54.8\%). In 
Table I. Cytoplasmic and nuclear expression of HuR in prostate carcinoma and normal prostate parenchyma.

\begin{tabular}{lccc}
\hline & $\begin{array}{c}\text { Normal tissue } \\
(\mathrm{n}=104)\end{array}$ & $\begin{array}{c}\text { Carcinoma } \\
(\mathrm{n}=104)\end{array}$ & $\begin{array}{c}\text { p-value } \\
\text { (Fisher's } \\
\text { exact test) }\end{array}$ \\
\hline $\begin{array}{l}\text { Cytoplasmic HuR } \\
\text { expression }\end{array}$ & & & \\
$\quad \begin{array}{l}\text { Negative } \\
\text { Positive }\end{array}$ & $103(99.0 \%)$ & $85(81.7 \%)$ & \\
Nuclear HuR & $1(1.0 \%)$ & $19(18.3 \%)$ & \\
expression & & & \\
$\quad$ Negative & & & 0.0005 \\
Positive & $17(16.3 \%)$ & $29(27.9 \%)$ & \\
\hline
\end{tabular}

cancer both an IRS of 8 and 12 was found in 35 cases (33.7\%). Thus, although nuclear HuR positivity was found in the majority of carcinomas $(72.1 \%)$ and normal tissue cases
(83.7\%), nuclear HuR expression was reduced in a subset of carcinomas compared to normal tissue with a borderline significance ( $p=0.065$, Table I). No correlation was detected between nuclear and cytoplasmic HuR immunoreativity scores in cancer (Spearman-rho, data not shown). Consistent with its reported role of a ubiquitously expressed protein, HuR staining was also identified in stromal fibroblasts and leiomyocytes, but was restricted to the nuclei in these cells.

Correlation of HuR expression with clinicopathological parameters. Prostate carcinomas can be described by a variety of clinical and pathological factors, which help to estimate biological behavior and prognosis of an individual tumor. We investigated whether HuR expression differed between tumors with certain characteristics. As shown in Table II, neither cytoplasmic nor nuclear HuR expression were associated with established prognostic factors such as Gleason score or tumor stage, and there was no association with patient age or resection margin status $(\mathrm{p}>0.5)$.

Survival analysis. In prostate cancer, an increase of serum PSA is a surrogate marker for disease relapse. To evaluate the prognostic implication of HuR expression in prostate cancer, we performed a univariate survival analysis determining PSA-

Table II. Association of cytoplasmic and nuclear HuR expression with clinicopathological factors.

\begin{tabular}{|c|c|c|c|c|c|c|c|}
\hline Characteristic & $\begin{array}{c}\text { All } \\
\text { cases }\end{array}$ & $\begin{array}{c}\text { HuR } \\
\text { cytoplasmatic } \\
\text { negative }(\%)\end{array}$ & $\begin{array}{c}\text { HuR } \\
\text { cytoplasmatic } \\
\text { positive }(\%)\end{array}$ & p-value & $\begin{array}{c}\text { HuR } \\
\text { nuclear } \\
\text { negative }(\%)\end{array}$ & $\begin{array}{c}\text { HuR } \\
\text { nuclear } \\
\text { positive }(\%)\end{array}$ & p-value \\
\hline Age & & & & $1.000^{\mathrm{a}}$ & & & $0.271^{\mathrm{a}}$ \\
\hline$\leq 63$ years & 60 & $49 \quad(81.7)$ & $11(18.3)$ & & $14(23.3)$ & $46 \quad(76.7)$ & \\
\hline$>63$ years & 44 & $36 \quad(81.8)$ & $8(18.2)$ & & $15(34.1)$ & $29(65.9)$ & \\
\hline $\mathrm{pT}$ & & & & $0.675^{\mathrm{b}}$ & & & $0.799^{b}$ \\
\hline pT2 & 62 & 52 (83.9) & $10(16.1)$ & & $18(29.0)$ & $44(71.0)$ & \\
\hline pT3 & 41 & $32(78.0)$ & $9(22.0)$ & & $11(26.8)$ & $30 \quad(73.2)$ & \\
\hline pT4 & 1 & $1(100.0)$ & $0 \quad(0.0)$ & & $0 \quad(0.0)$ & $1(100.0)$ & \\
\hline Gleason score & & & & $0.177^{\mathrm{b}}$ & & & $0.627^{\mathrm{b}}$ \\
\hline $2-6$ & 42 & 37 (88.1) & $5(11.9)$ & & $10(23.8)$ & 32 (76.2) & \\
\hline 7 & 36 & $26(72.2)$ & $10(27.8)$ & & $10(27.8)$ & $26(72.2)$ & \\
\hline $8-10$ & 26 & $22(84.6)$ & $4(15.4)$ & & $9(34.6)$ & $17(65.4)$ & \\
\hline Resection margin & & & & $0.320^{\mathrm{a}}$ & & & $0.186^{\mathrm{a}}$ \\
\hline R0 & 60 & $47 \quad(78.3)$ & $13(21.7)$ & & $20(33.3)$ & $40 \quad(66.7)$ & \\
\hline $\mathrm{R} 1$ & 44 & 38 (86.4) & $6(13.6)$ & & $9(20.5)$ & 35 (79.5) & \\
\hline \multicolumn{8}{|l|}{ Pre-operative } \\
\hline $\operatorname{PSA}(n=96)$ & & & & $1.000^{\mathrm{a}}$ & & & $1.000^{\mathrm{a}}$ \\
\hline$\leq 8.6 \mathrm{ng} / \mathrm{ml}$ & 48 & 39 (81.3) & $9(18.7)$ & & $13(27.1)$ & 35 (72.9) & \\
\hline$>8.6 \mathrm{ng} / \mathrm{ml}$ & 48 & $39 \quad(81.3)$ & $9(18.7)$ & & $14(29.2)$ & $34(70.8)$ & \\
\hline
\end{tabular}

${ }^{\mathrm{a}}$ Fisher's exact test. ${ }^{\mathrm{b}} \chi^{2}$ test. 
Table III. Univariate survival analysis (Kaplan-Meier) and multivariate Cox regression analysis (variables remaining in the equation).

Univariate survival analysis

Characteristic

No. of cases $\quad$ No. of events
Mean survival time in months (SE)

p-value

(log-rank test)

\section{Age at diagnosis}

$\leq 63$ years

$>63$ years

Cytoplasmatic HuR expression

\section{Negative}

Positive

Nuclear HuR expression

Negative

Positive

pT

$$
\text { pT2 }
$$

pT3-4

Gleason score

2-6

7

$8-10$
60

44

85

19

29

75

62

42

42

36

26
0.258

65.7 (5.2)

$71.2(5.0)$

0.843

$68.7(4.3)$

$69.9(9.3)$

7

0.040

$54.5(6.6)$

$73.8(4.4)$

23

0.007

$78.4(4.9)$

56.5 (5.9)

21

0.041

$75.8(6.0)$

$69.2(5.7)$

$49.1(5.6)$

Resection margin

R0 60

$60 \quad 19$

0.593

R1

44

17

$71.7(5.3)$

$61.4(5.0)$

Pre-operative PSA ( $\mathrm{n}=96)$

$\leq 8.6 \mathrm{ng} / \mathrm{ml}$

$48 \quad 14$

$14 \quad 76.3(5.5)$

$>8.6 \mathrm{ng} / \mathrm{ml}$

$48 \quad 21$

$58.5(6.0)$

Multivariate survival analysis

Characteristic

Hazard ratio

$95 \%$ confidence interval

p-value

Nuclear HuR expression

$0.229-0.965$

0.040

Negative

1.00

Positive

0.471

pT (pT2 vs. pT3-4)

pT2

$1.427-5.663$

0.003

pT3-4

1.00

2.843

Pre-operative PSA $(\mathrm{n}=96)$

$\leq 8.6 \mathrm{ng} / \mathrm{ml}$

1.1052-4.171

0.035

1.00

2.095

relapse-free survival time (Table III). We found nuclear HuR expression to be a favorable prognostic factor for disease-free survival time. Thus, patients whose tumors showed nuclear HuR staining had a longer mean disease-free survival time compared to patients with tumors lacking nuclear $\mathrm{HuR}$ expression (73.8 vs. 54.5 months, 5 -year survival rate $62.7 \%$ vs. $50.1 \% \mathrm{p}=0.04$, Fig. 2A). Survival time did not differ between patients with negative or positive cytoplasmic HuR staining 


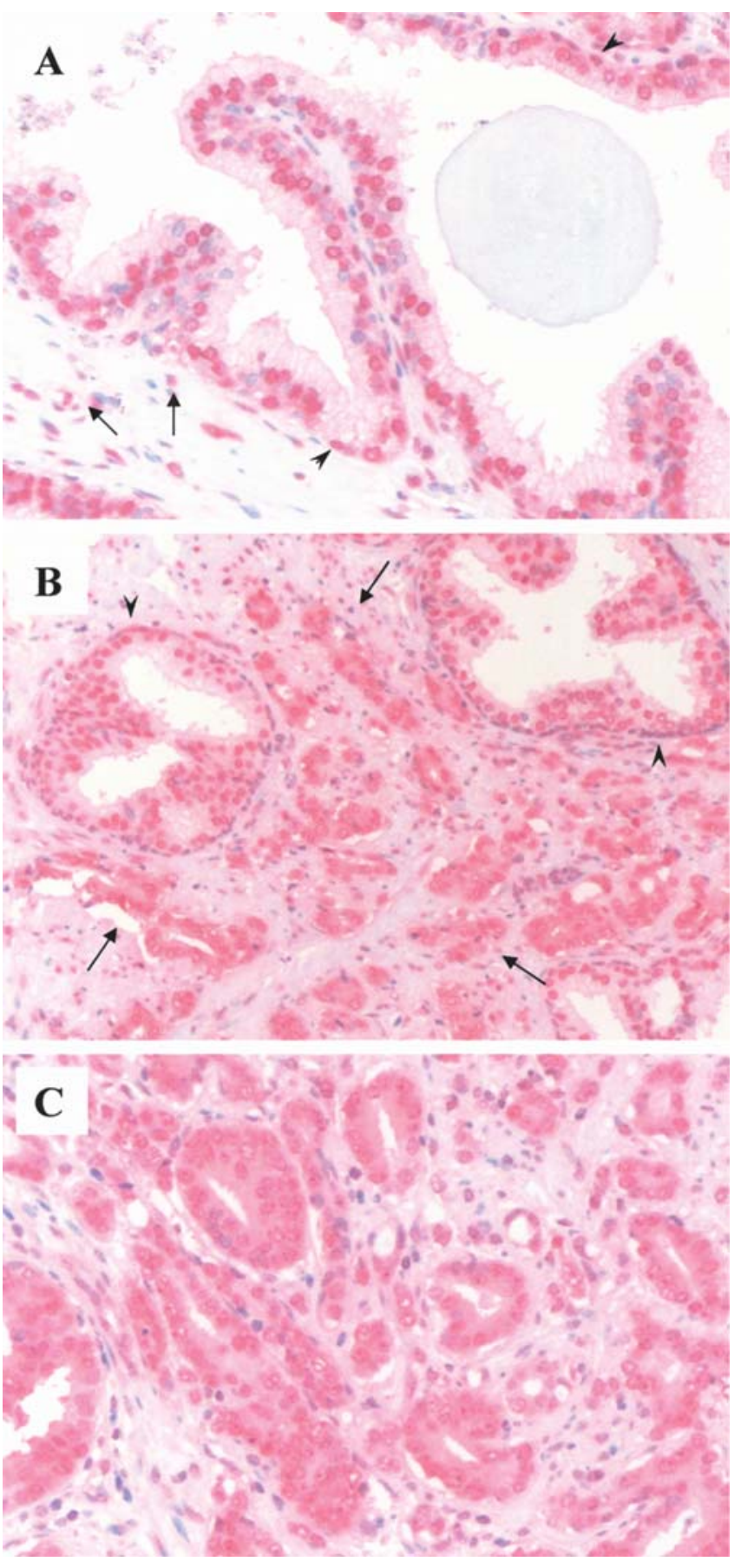

Figure 1. HuR immunoreactivity in human prostate carcinoma. A, Normal prostate glands with nuclear HuR positivity in secretory and basal epithelium (arrowheads). The cytoplasmic staining signal is very faint. Note stromal cells with HuR-positive nuclei (arrows). B, Strong nuclear and cytoplasmic immunoreactivity for HuR in infiltrating glands of a poorly differentiated prostate adenocarcinoma (arrows). Residual normal prostate glands display nuclear HuR positivity (arrowheads). C, High power view of a poorly differentiated prostate carcinoma with strong nuclear and cytoplasmic HuR expression.

of their respective tumors. Tumor stage, Gleason score [grouped according to Bostwick et al (17)], and pre-operative PSA were significant prognostic indicators in our study group as well (Table III and Fig. 2B).

We subsequently determined if nuclear HuR expression might be an independent prognostic factor and included it in an exploratory multivariate Cox regression analysis. In
A
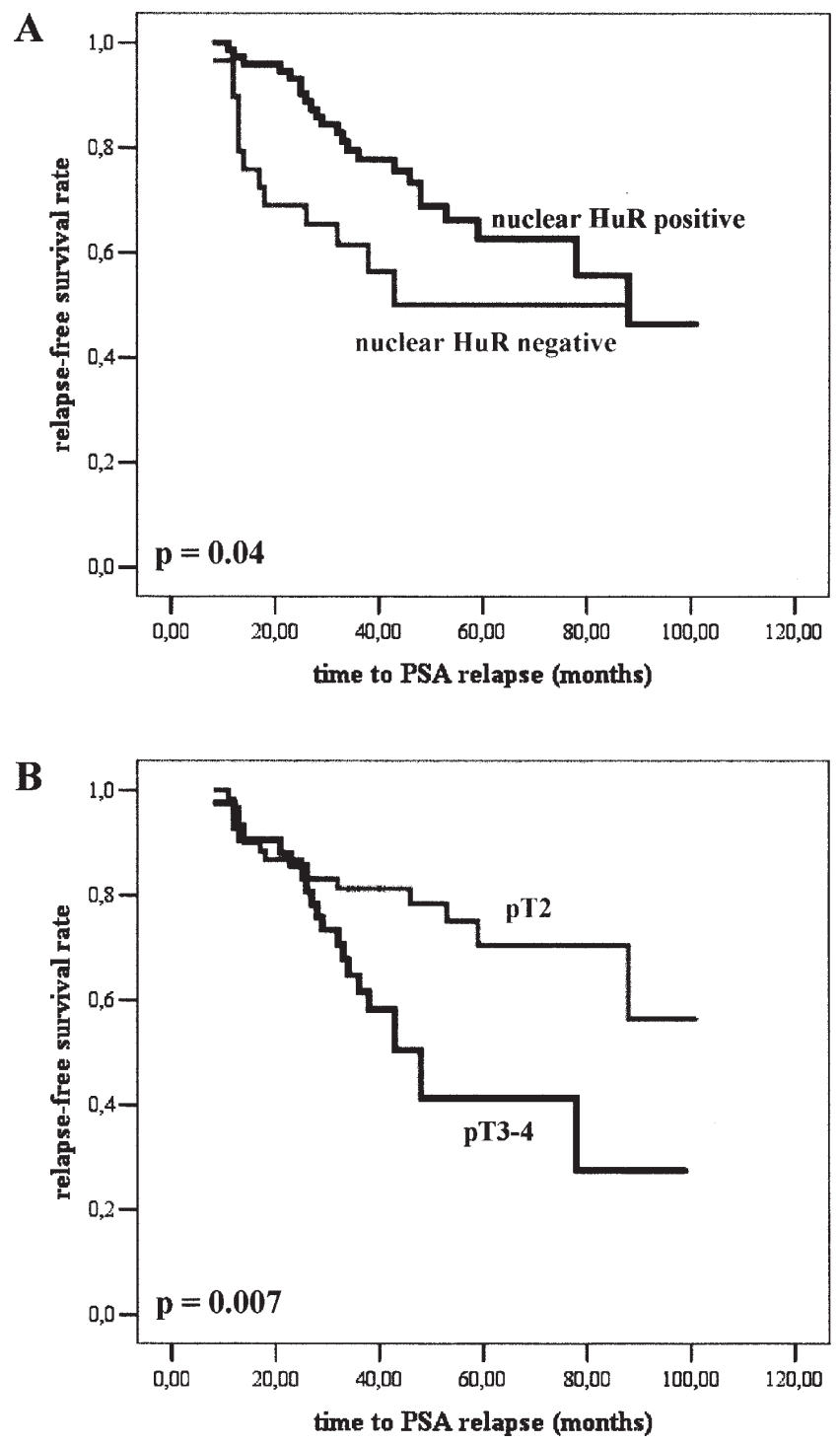

Figure 2. Kaplan-Meier analysis demonstrating the impact of nuclear expression of HuR and tumor stage on relapse-free survival of prostate carcinoma patients. Patients whose tumors displayed low nuclear HuR expression (A) or were locally advanced (B) had a shorter disease-free survival time. P, pvalue, log-rank test.

addition to the other significant prognostic markers, tumor stage, Gleason score and pre-operative PSA, we included age as well. As shown in Table III, nuclear HuR expression remained a significant prognostic factor in this setting $(\mathrm{p}=0.04)$. The relative risk of disease relapse in patients whose tumors showed nuclear HuR expression was less than half the risk of patients with carcinomas negative for nuclear HuR (hazard ratio $=0.471)$. Further on, tumor stage and pre-operative PSA were an independent prognostic marker in our study population (Table III).

Correlation of HuR and COX-2 expression. As HuR is known to stabilize COX-2 mRNA and to enhance COX-2 protein expression in vitro, we investigated whether this relationship was reflected in tumor tissue in vivo. We detected a significant correlation of moderate strength between cytoplasmic HuR and COX-2 immunoreactivity scores (correlation coefficient 0.27 (Spearman-rho), $\mathrm{p}=0.005$ ), and thereby showed that 
A
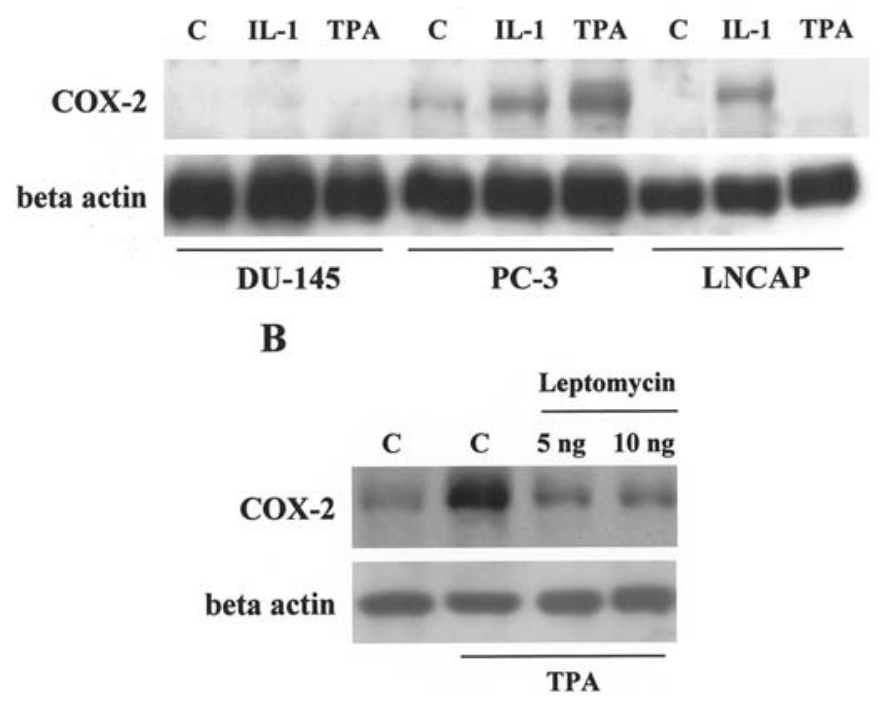

Figure 3. Immunoblot analysis of COX-2 expression in prostate cancer cell lines. Basal as well as inducible COX-2 expression in DU-145, PC-3, and LNCAP prostate cancer cells. C, unstimulated control (A). Reduction of inducible COX-2 expression by Leptomycin B treatment in PC-3 cells. C, untreated control (B).

positivity for cytoplasmic HuR was paralleled by an increased expression of the COX-2 protein in prostate carcinoma (data not shown). Nuclear HuR expression was not correlated with COX-2 expression (Spearman-rho, data not shown).

Induction of COX-2 in prostate cancer cells. To study the expression of COX-2 in a cell culture model, we treated the prostate cancer cell lines DU-145, PC-3, and LNCAP with IL-1, and TPA. A weak constitutive COX-2 expression was found in PC-3 cells. In these cells, COX-2 expression could be markedly enhanced by treatment with TPA, and a moderate increase of COX-2 expression was seen after IL-1, treatment (Fig. 3A). In LNCAP and DU-145 cells, no constitutive COX-2 expression was observed, but a moderate induction of COX-2 could be achieved by IL-1 stimulation in LNCAP cells (Fig. 3A).

Effect of HuR inhibition on COX-2 expression. To determine whether COX-2 expression might be dependent on the expression of $\mathrm{HuR}$ in prostate cancer, we inhibited HuR pharmacologically. We used TPA-stimulated PC-3 cells in which COX-2 induction was strongest, and applied Leptomycin B, an indirect HuR inhibitor. The antiproliferative antibiotic Leptomycin B reduces HuR activity by inhibiting CRM1, a nuclear export protein essential for the nucleocytoplasmic shuttling of HuR ligands (pp32 and APRIL), which are required for HuR activity (18). As shown in Fig. 3B, the induction of COX-2 protein expression was supressed after Leptomycin-treatment in TPA-stimulated cells.

\section{Discussion}

In this study we systematically compared the expression of the human ELAV-like protein HuR in normal and neoplastic prostate tissue. We detected an overexpression of cytoplasmic and a relative loss of nuclear HuR expression in cancer, and found this lack of nuclear HuR expression to be independently linked to a reduced relapse-free patient survival. Investigating COX-2 as a known target of the mRNA stabilizing activity of HuR, we observed a significant positive association between COX-2 expression in tumor tissue and HuR immunoreactivity.

To our knowledge this is the first study to determine the expression pattern of $\mathrm{HuR}$ in a large cohort of wellcharacterized prostate carcinomas. The results are in line with our previous findings of a cytoplasmic overexpression of HuR in breast (5), ovarian (7), and colorectal cancer (6), and indicate that subcellular localization of HuR is deregulated in a subset of prostate cancers. The functional consequences of this deregulation are explicable by the role of HuR as a nuclear shuttling protein. HuR binds to its target mRNAs in the nucleus and is subsequently translocated to the cytoplasm, where mRNA translation into protein takes place (3). Several mRNA targets have been reported for HuR, for example the mRNAs of the angiogenic factor vascular endothelial growth factor (VEGF), the inflammatory cytokines interleukin-8 (IL-8), interleukin-6 (IL-6), and tumor necrosis factor $\alpha$ $(\mathrm{TNF}-\alpha)(19)$ as well as cell cycle regulatory proteins such as cyclin A and cyclin B1 (20). In addition, the mRNAs of the proto-oncogenes c-fos (4) and c-myc (21) as well as urokinase plasminogen activator (uPA) and uPA receptor (UPAR) (22) have been described as targets of HuR. It is thus conceivable that an increased RNA-stabilizing function of HuR might enhance the expression of proteins that are involved in angiogenesis, tumor-associated inflammation and cellular growth. These mechanisms are also discussed to be causative for the observation of an adverse prognostic impact of altered cellular localization of HuR, which has been described in ovarian $(7,23)$, breast $(24)$, and gastric carcinoma (25). In these neoplasms cytoplasmic HuR expression was shown to be prognostic of patient survival. In the present investigation we additionally showed that an imbalance of the HuR nuclear export system can be observed on the level of nuclear HuR, as well.

The association of HuR and COX-2 expression in solid tumors has been reported by our group and others in breast (5), ovarian (7,23), gastric (25), and colorectal cancer (6). Additionally, functional studies have shown that binding of COX-2 mRNA by HuR results in an enhanced expression of the COX-2 protein. Thus, transfection of human LoVo colon cancer cells with a HuR expression vector resulted in constitutive COX-2 expression as well as increased VEGF and IL-8 expression (8). In this study we provided evidence that COX-2 overexpression in prostate carcinoma is due to deregulated HuR activity. Showing that HuR inhibition is paralleled by a reduced expression of COX-2 in vitro and the observation of a prognostic impact of nuclear HuR deficiency in vivo, our data suggest the further evaluation of $\mathrm{HuR}$ as an attractive novel target in prostate cancer therapy. Similar to us, Erkinheimo et al have shown that Leptomycin B reduced COX-2 expression in IL-1 stimulated OVCAR-3 ovarian cancer cells (23). In LNCAP and DU-145 prostate cancer cells, this agent further induced cell cycle arrest and apoptosis as well as induction of p53 (26). Further evaluation of Leptomycin B analogs as indirect inhibitors of HuR would be 
intriguing in cancers with deregulated HuR such as prostate carcinoma.

Several mechanisms controlling the subcellular localization of HuR have been identified to date. The cytoplasmic translocation of HuR is inhibited by activated AMP-activated kinase (AMPK) (27). This enzyme functions as an energy sensor in the cell and provides metabolic adaptation under ATP-depleting conditions. Interestingly, AMPK activation has been shown to display tumor-suppressing functions by inhibiting the proliferation of DU-145, PC-3, and LNCAP prostate cancer cells (28), otherwise AMPK activation has also been shown to stabilize the mRNA of VEGF thereby enhancing the angiogenic potential of DU-145 prostate cancer cells (29). In the context of our findings, it would be interesting to determine the interaction between AMPK and HuR and their impact on the tumorigenic properties of prostate cancer cells. Another molecule potentially relevant in HuR function in prostate cancer is pp32, a nuclear phosphoprotein, which forms a complex with HuR and the SET protein, resulting in enhanced mRNA-stabilizing activity of HuR (2). pp32 is frequently overexpressed in prostate carcinomas and seems to contribute to a less differentiated phenotype of the tumor cells (30).

In conclusion, our expression data demonstrate a derangement of the subcellular localization of HuR in prostate cancer, which is linked to altered expression of COX-2 and adverse patient prognosis. Based on our results, further studies should evaluate the molecular network controlled by HuR and controlling HuR to gain further insight into the value of $\mathrm{HuR}$ as a potential new target in prostate cancer therapy. Additionally, the prognostic role of HuR expression should be confirmed in large-scale clinical trails.

\section{Acknowledgements}

We thank Ms. Ines Koch for her excellent technical assistance. This study was supported by a grant of the Berliner Krebsgesellschaft.

\section{References}

1. Jemal A, Siegel R, Ward E, Murray T, Xu J and Thun MJ: Cancer statistics 2007. CA Cancer J Clin 57: 43-66, 2007.

2. Brennan CM and Steitz JA: HuR and mRNA stability. Cell Mol Life Sci 58: 266-277, 2001.

3. Atasoy U, Watson J, Patel D and Keene JD: ELAV protein HuA (HuR) can redistribute between nucleus and cytoplasm and is upregulated during serum stimulation and $\mathrm{T}$ cell activation. J Cell Sci 111: 3145-3156, 1998.

4. Peng SS, Chen CY, Xu N and Shyu AB: RNA stabilization by the AU-rich element binding protein, HuR, an ELAV protein. EMBO J 17: 3461-3470, 1998.

5. Denkert C, Weichert W, Winzer KJ, et al: Expression of the ELAV-like protein HuR is associated with higher tumor grade and increased cyclooxygenase- 2 expression in human breast carcinoma. Clin Cancer Res 10: 5580-5586, 2004.

6. Denkert C, Koch I, von Keyserlingk N, et al: Expression of the ELAV-like protein HuR in human colon cancer: association with tumor stage and cyclooxygenase-2. Mod Pathol 19: 1261-1269, 2006.

7. Denkert C, Weichert W, Pest S, et al: Overexpression of the embryonic-lethal abnormal vision-like protein HuR in ovarian carcinoma is a prognostic factor and is associated with increased cyclooxygenase 2 expression. Cancer Res 64: 189-195, 2004.

8. Dixon DA, Tolley ND, King PH, et al: Altered expression of the mRNA stability factor HuR promotes cyclooxygenase-2 expression in colon cancer cells. J Clin Invest 108: 1657-1665, 2001 .
9. Sengupta S, Jang BC, Wu MT, Paik JH, Furneaux H and Hla T: The RNA-binding protein HuR regulates the expression of cyclooxygenase-2.J Biol Chem 278: 25227-2533, 2003.

10. O'Banion MK, Winn VD and Young DA: cDNA cloning and functional activity of a glucocorticoid-regulated inflammatory cyclooxygenase. Proc Natl Acad Sci USA 89: 4888-4892, 1992.

11. Fujita H, Koshida K, Keller ET, et al: Cyclooxygenase-2 promotes prostate cancer progression. Prostate 53: 232-240, 2002.

12. Denkert C, Thoma A, Niesporek S, et al: Overexpression of cyclooxygenase- 2 in human prostate carcinoma and prostatic intraepithelial neoplasia-association with increased expression of Polo-like kinase-1. Prostate 67: 361-369, 2007.

13. Madaan S, Abel PD, Chaudhary KS, et al: Cytoplasmic induction and over-expression of cyclooxygenase- 2 in human prostate cancer: implications for prevention and treatment. BJU Int 86: 736-741, 2000.

14. Cohen BL, Gomez P, Omori Y, et al: Cyclooxygenase-2 $(\mathrm{COX}-2)$ expression is an independent predictor of prostate cancer recurrence. Int J Cancer 119: 1082-1087, 2006.

15. Rubio J, Ramos D, Lopez-Guerrero JA, et al: Immunohistochemical expression of Ki-67 antigen, cox-2 and $\mathrm{Bax} / \mathrm{Bcl}-2$ in prostate cancer; prognostic value in biopsies and radical prostatectomy specimens. Eur Urol 48: 745-751, 2005.

16. Denkert C, Kobel M, Pest S, et al: Expression of cyclooxygenase 2 is an independent prognostic factor in human ovarian carcinoma. Am J Pathol 160: 893-903, 2002.

17. Bostwick DG, Grignon DJ, Hammond ME, et al: Prognostic factors in prostate cancer. College of American Pathologists Consensus Statement 1999. Arch Pathol Lab Med 24: 995-1000, 2000.

18. Brennan CM, Gallouzi IE and Steitz JA: Protein ligands to HuR modulate its interaction with target mRNAs in vivo. J Cell Biol 151: 1-14, 2000.

19. Nabors LB, Gillespie GY, Harkins L and King PH: HuR, a RNA stability factor, is expressed in malignant brain tumors and binds to adenine- and uridine-rich elements within the $3^{\prime}$ untranslated regions of cytokine and angiogenic factor mRNAs. Cancer Res 61: 2154-2161, 2001.

20. Wang W, Caldwell MC, Lin S, Furneaux H and Gorospe M: HuR regulates cyclin $A$ and cyclin $B 1$ mRNA stability during cell proliferation. EMBO J 19: 2340-2350, 2000.

21. Lafon I, Carballes F, Brewer G, Poiret M and Morello D: Developmental expression of AUF1 and HuR, two c-myc mRNA binding proteins. Oncogene 16: 3413-3421, 1998.

22. Tran H, Maurer F and Nagamine Y: Stabilization of urokinase and urokinase receptor mRNAs by HuR is linked to its cytoplasmic accumulation induced by activated mitogen-activated protein kinase-activated protein kinase 2. Mol Cell Biol 23: 7177-7188, 2003.

23. Erkinheimo TL, Lassus H, Sivula A, et al: Cytoplasmic HuR expression correlates with poor outcome and with cyclooxygenase 2 expression in serous ovarian carcinoma. Cancer Res 63: 7591-7594, 2003.

24. Heinonen M, Bono P, Narko K, et al: Cytoplasmic HuR expression is a prognostic factor in invasive ductal breast carcinoma. Cancer Res 65: 2157-2161, 2005.

25. Mrena J, Wiksten JP, Thiel A, et al: Cyclooxygenase-2 is an independent prognostic factor in gastric cancer and its expression is regulated by the messenger RNA stability factor HuR. Clin Cancer Res 11: 7362-7368, 2005.

26. Lecane PS, Kiviharju TM, Sellers RG and Peehl DM: Leptomycin B stabilizes and activates p53 in primary prostatic epithelial cells and induces apoptosis in the LNCaP cell line. Prostate 54: 258-267, 2003.

27. Wang W, Fan J, Yang X, et al: AMP-activated kinase regulates cytoplasmic HuR. Mol Cell Biol 22: 3425-3436, 2002.

28. Xiang X, Saha AK, Wen R, Ruderman NB and Luo Z: AMPactivated protein kinase activators can inhibit the growth of prostate cancer cells by multiple mechanisms. Biochem Biophys Res Commun 321: 161-167, 2004.

29. Yun H, Lee M, Kim SS and Ha J: Glucose deprivation increases mRNA stability of vascular endothelial growth factor through activation of AMP-activated protein kinase in DU145 prostate carcinoma. J Biol Chem 280: 9963-9972, 2005.

30. Brody JR, Kadkol SS, Hauer MC, Rajaii F, Lee J and Pasternack GR: pp32 reduction induces differentiation of TSU-Pr1 cells. Am J Pathol 164: 273-283, 2004. 